\title{
RaPID ANd Automatic ATlas-based APPROACh Of AlZheimer's Disease AsSessment By Positron Emission Tomography NeUroimageS
}

\author{
Guoyu Qian ${ }^{1}$, Xuezhen Shen $^{2}$, Suhuai Luo ${ }^{1}$, Jesse S. Jin ${ }^{1}$, Wieslaw L. Nowinski ${ }^{3}$ \\ ${ }^{1}$ School of Design, Communication and IT, The University of Newcastle, Australia \\ ${ }^{2}$ Shanghai Lixin University of Commerce, China \\ ${ }^{3}$ Biomedical Imaging Lab, Agency for Science, Technology and Research, Singapore
}

\begin{abstract}
Current Alzheimer's disease diagnosis and cognitive assessment are based on medical history assessment and evaluation of cognitive score systems. They are time-consuming and subjective. A rapid and automated method is developed by processing positron emission tomography neuroimages and performing statistical analysis. The brain areas are firstly extracted from the neuroimages by an atlas-assisted approach, and then transformed piecewise into a common atlas space by dividing the brain into 18 cubic regions based on the landmarks identified automatically. The statistical models of stepwise regressions and discriminant classification are applied to predict the cognitive scores and make a diagnosis on Alzheimer's disease or mild cognitive impairment. The proposed method is fully automatic and has been tested on 400 cases. The preliminary testing results are promising. For a group of 250 cases which are the samples of the regressions and discriminant classification, the success rates of disease diagnosis are $73.7 \%, 54.9 \%$, and $79.7 \%$ for the patients with Alzheimer's disease, mild cognitive impairment, and normal subjects, respectively. The average success rate for another group of 150 cases is $61.3 \%$.
\end{abstract}

Keywords: image processing, brain atlas, biostatistics

\section{INTRODUCTION}

Alzheimer's disease (AD) is a major form of dementia and one of increasing neurodegenerative diseases. Mild cognitive impairment (MCI) is a diagnosis to those who have disorder of memory, language, or other mental functions, but do not interfere significantly with their daily life [1]. It may lead to $\mathrm{AD}$, or remain as MCI for many years. Currently the steps in the diagnosis process of $\mathrm{AD}$ and $\mathrm{MCI}$ are medical history assessment and evaluation of memory and thinking abilities. Several cognitive score systems, e.g. mini mental state examination (MMSE) [2], clinical dementia rating (CDR) assignment [3], are commonly used to evaluate the abilities of language, memory, orientation, etc. for patients. However, the conventional diagnosis is time-consuming and subjective, and needs professional knowledge as well.

The cognitive scores are correlated with the changes on structural and functional neuroimages of several brain structures or regions [4-7]. Neuroimaging techniques such as computed tomography (CT), magnetic resonance imaging (MRI), or positron emission tomography (PET), provide the possibilities to investigate the human brains in vivo by visual inspection or quantitative analysis of brain structures with computer technologies. CT and MRI are widely used imaging tools to discover and compare the structural changes and assist disease diagnose. However, for neurodegenerative diseases like $\mathrm{AD}$, the structural changes of an affected brain are too subtle to be visible or detected on the images of CT or MRI at the initial stage of the disease. PET provides the information about blood flow or metabolism during the scanning in any part of the brain. In a PET scan, the subject is injected with a very small quantity of radioactive glucose, fluorodeoxyglucose (FDG). The hypometabolism is observed in several regions of the brain with AD or MCI [8]. Brain cells use glucose as fuel, and if they are more active, they will consume more of the radioactive glucose, and if less active, they will consume less of it. Computer program uses the absorption data to show the levels of activity as a gray brain map, with one value (usually bright) indicating more active brain areas, and another value (usually dark) indicating the less active areas.

It is a challenging task to build a relationship quantitatively between the changes on neuroimages and the cognitive scores, due to the multiple impacts of cognitive scores such as language, orientation, etc. as well as the multiple factors of neurological changes of brain such as structure atrophy, brain cell hypometabolism, etc. For instance, the atrophy of the hippocampus may cause memory problem of a patient and have lower MMSE scores than those of normal subjects. But it is difficult to determine which factors in the MMSE score system and how many percent of the scores are affected. For a large number of cases which have both cognitive scores and the changes on neuroimages, statistical analysis is a feasible solution to build the correlation and establish the relationship between them by constructing several equations or functions.

In this paper, a rapid and automatic atlas-based approach of cognitive assessment and AD diagnosis is presented. It is based on a set of atlas-assisted neuroimage processing algorithms for the measurement of the brain activity changes, and the statistical analysis for the cognitive assessment and the disease diagnosis. A group of 250 cases was processed and 15 brain structures were extracted for statistical analysis. The regression equations were constructed from the stepwise regressions to assess the cognitive scores, and the discriminant functions were generated from the step of the discriminant classification to diagnose $\mathrm{AD}$ or $\mathrm{MCI}$. The promising results were given after verifying the regression equations and discriminant functions by another group of 150 cases.

\section{RELATED WORKS}

A linkage between the dementia and certain regions of brain was demonstrated by many research papers [4-7]. As the most common form of dementia in the elderly, the AD patients have significant lower regional metabolism than the healthy subjects in the region of the temporal lobes in both left and

\footnotetext{
Proc. 9th IEEE Int. Conf. on Cognitive Informatics [ICCl'10] F. Sun, Y. Wang, J. Lu, B. Zhang, W. Kinsner \& L.A. Zadeh [Eds.] 978-1-4244-8040-1/10/\$26.00 @2010 IEEE
} 
right hemispheres. Measurement of glucose metabolism reduction from FDG-PET images is becoming a standard technique to predict or diagnose $\mathrm{AD}$ and MCI [8, 9]. The temporal lobe is reported as one of the first regions to be affected by $\mathrm{AD}$, and confirmed and verified pathologically [10]. However, several areas such as cerebellum are reported less affected by AD [11].

As neuroimages are typically very large (millions of voxels), the data reduction methods are applied to reduce the computational cost before the statistical analysis. They are roughly divided into two groups: voxel-based methods and region-of-interest (ROI) based methods. The former considers each voxel as a separate entity for statistical analysis, and the latter focuses on particular areas of the brain to summarize the massive amounts of data in an area into a single number for each ROI in the brain, for example, the average value of all voxels within the region. The most commonly cited software packages for performing voxel-based analysis are statistical parametric mapping (SPM) [12] and FMRIB software library (FSL) [13]. The methods are typically applied to the applications which need the analysis of whole brain, but they are usually computational costly than that of ROI-based approaches. It may face difficulties to study accurately small regions such as hippocampus in atrophy brains. Since the linkage between $\mathrm{AD}$ and certain areas of the brain is commonly recognized, the ROI-based methods are more efficient for statistical analysis. They are applied to both structural medical images [14] and functional medical images [15]. These summarized values generated by ROI-based methods may then be used in statistical analysis such as analysis of variance (ANOVA) and regression.

One of the ROI-based methods is atlas-assisted brain structure extraction from neuroimages. Atlas-assisted operations on medical images of human brain are widely applied to image segmentation, data normalization, and localization analysis [16-18], especially for the PET images with a poorer spatial resolution and lower signal-noise ratio than other modalities like CT or MRI. Talairach atlas [19] is widely used in clinical applications for spatial normalization and registration of images from different patients or even different modalities. It is commonly used as a reference brain and a gold standard in human brain mapping. A set of modified Talairach landmarks, conceptually equivalent to the original Talairach landmarks, was introduced [20] to overcome the problems and limitations of the original landmarks and become more constructive and easier identification by computer program. It is extended to include the cerebellum of a brain into the Talairach space as well [21].

The statistical analysis was performed to analyze the relationship between the $\mathrm{AD}$ diagnosis and the different score systems like MMSE and intelligence quotient [6], the relationship between the cognitive function and regional cerebral flow [22], and the correlations between cerebral glucose metabolism and neuropsychological test [23]. However, the statistical analysis was only used as a discovering tool to confirm the relationships or correlations between the cognitive scores and the changes of the neuroimages. It was rarely applied to generate the equations or functions for cognitive scores based on the changes detected from the neuroimages.

\section{MATERIALS}

The proposed approach was evaluated with a total of 400 experiment subjects as well as a high resolution threedimension brain atlas.

\section{A. Experiment Subjects}

The experiment subjects were downloaded from the website of Alzheimer's Disease Neuroimaging Initiative (ADNI) (http://www.loni.ucla.edu/ADNI). Each case includes the personal information, cognitive scores, diagnosis result, and FDG-PET volumetric images.

\section{1) Personal Information}

The personal information contains the patient identity (ID), date of birth, date of study, gender, etc. Only the information of gender and age is included in our study. There are 274 males and 126 females; their average age at study day is 76.9 years old, and the standard deviation of the ages is 6.5 years old, i.e. $76.9 \pm 6.5$.

\section{2) Cognitive Scores}

Most of the subjects were evaluated with several cognitive score systems including MMSE, CDR, functional assessment questionnaire (FAQ), and neuropsychiatric inventory (NPI). The MMSE scores $(26.3 \pm 3.5)$ and CDR scores $(0.5 \pm 0.4)$ are included into our study because of their widespread use in clinical diagnosis of $\mathrm{AD}$ and $\mathrm{MCI}$.

\section{3) Diagnosis Results}

All of the subjects had been clinically assessed as cognitively normal (122 subjects or $30.5 \%)$ or diagnosed with $\mathrm{AD}$ (66 subjects or $16.5 \%)$ or MCI (212 subjects or $53 \%)$. The evaluation of the proposed methods is based on these assessment and diagnosis results as gold standard.

\section{4) FDG-PET Images}

The FDG-PET images are 6 dynamic frames with scanning every 5 minutes from 30 to 60 minutes after FDG injection. Those scans were preprocessed by the following steps: 1) registered to the first frame, 2) averaged, 3) reoriented into a grid of $160 \times 160 \times 96$ voxels with the voxel size $1.5 \times 1.5 \times 1.5 \mathrm{~mm}$ [24]. The preprocessed images have their horizontal axis paralleled with the anterior commissure and posterior commissure. Fig. 1 shows the examples of images from a patient with MCI. Fig. 1a is a slice of the original baseline images; Fig. 1b is another original image co-registered with baseline images; Fig. 1c is an averaged image of 6 dynamic scans; and Fig. 1d is a slice after reorientation.

\section{B. AD-specific Brain Atlas}

The brain atlas was constructed from a high quality MRI volumetric data with the brain structures labeled, including some $\mathrm{AD}$-specific structures like the hippocampus, amygdala, etc. [25]. It is a high resolution volumetric dataset of $512 \times 512 \times 211$ voxels with $0.32 \times 0.32 \times 0.6 \mathrm{~mm}^{3}$. Fig. 2 gives several examples of the atlas warping on the PET images. Fig. $2 a$ and $2 b$ show the atlas images on two axial slices of PET images with label texts. Fig. $2 c$ and $2 d$ are the integrations of 
atlas contours on a coronal slice and a sagittal slice, respectively.
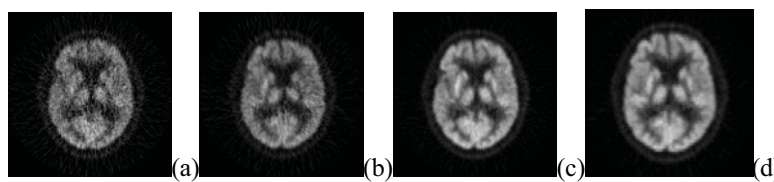

Figure 1. ADNI images: (a) a slice of original baseline images; (b) coregistered image; (c) averaged image of dynamic scans; (d) reoriented image.
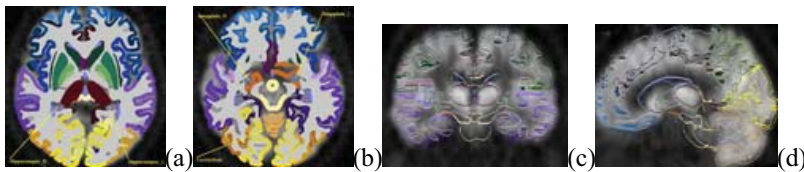

Figure 2. Atlas warping on FDG-PET images: (a)(b) atlas images on axial slices with label texts; (c) atlas contours on coronal slice; (d) atlas contours on sagittal slice

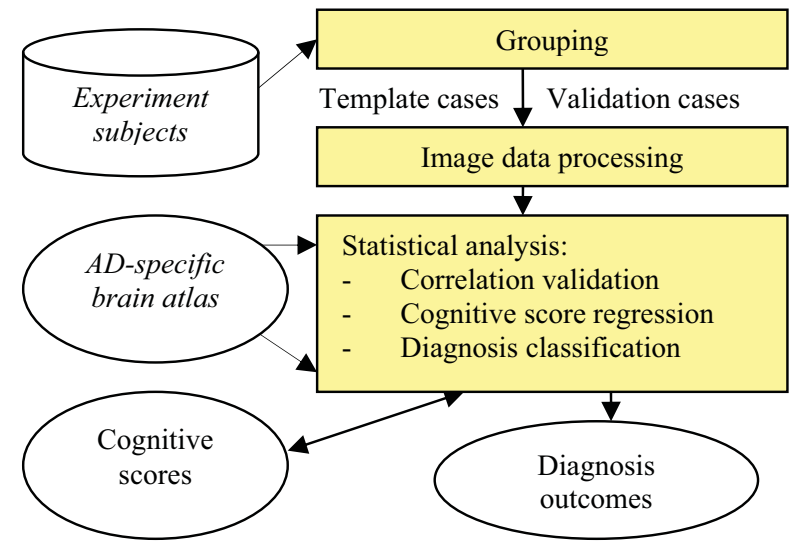

Figure 3. Flowchart of cognitive assessment and AD/MCI diagnosis

\section{METHODS}

Fig. 3 shows the flowchart of the proposed approach. It is an atlas-assisted ROI approach to automatically extract the AD-specific structures from brain images for statistical analysis by making use of the FDG-PET images and the AD-specific brain atlas. It consists of the following components: grouping of template cases and validation cases, atlas-based image data processing, and statistical analysis.

\section{A. Data Grouping}

All cases are randomly divided into two groups: template (GTL) and validation (GVD), as listed in Table I. The GTL cases are used to generate the regression equations for MMSE and CDR by the statistical model of stepwise regression based on the glucose metabolism values extracted from the ADspecific structures of FDG-PET images. In addition, the GTL cases are also applied to generate the discriminant functions by the statistical model of discriminant classification based on the score values of MMSE or CDR. The functions are calculated on each case for the classification of the groups: AD, MCI, or Normal (NL). The GVD cases are for testing purpose only to verify the regression equations and discriminant functions.
TABLE I. GROUPS OF TEMPLATE AND VALIDATION CASES

\begin{tabular}{|l|l|l|l|l|}
\hline & Total & AD (\%) & MCI (\%) & NL (\%) \\
\hline GTL & 250 & $38(15.2)$ & $133(53.2)$ & $79(31.6)$ \\
\hline GVD & 150 & $28(18.7)$ & $79(52.7)$ & $43(28.7)$ \\
\hline Total & 400 & $66(30.5)$ & $212(53)$ & $122(16.5)$ \\
\hline
\end{tabular}

\section{B. Image Data Processing}

This component is to extract the glucose metabolism information from the brain images for statistical analysis. It consists of two different types of normalization: spatial normalization and intensity normalization. The former is to transform the images to a common atlas space by several steps of image processing, and the latter is to adjust the range of intensity values by dividing the value with that of the cerebellum, which is the less-affected area by the disease.
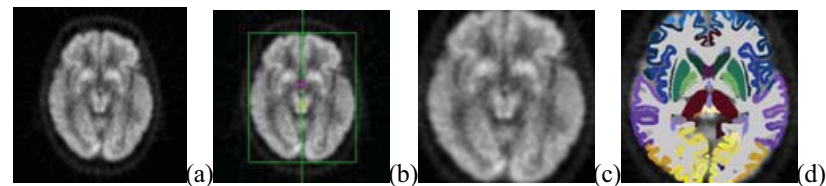

Figure 4. An example of spatial normalization steps: (a) original axial slice; (b) slice with landmarks identified; (c) slice transformed to the atlas space; (d) transformed slice with atlas warped.

The spatial normalization plays the role to process the PET images and transform them into the atlas space. It consists of three steps: 1) the brain areas are segmented from the PET images by a threshold which is selected by a histogram graph automatically [26], 2) the set of landmarks is accurately defined in the atlas space and automatically detected on PET images [21], and 3) based on the landmarks, the brain is divided into 18 cubic regions and a piecewise linear transformation is applied to generate a new volumetric image data fitting into the atlas space. Fig. 4 shows an example of the results after spatial normalization. Fig. $4 \mathrm{a}$ is an original axial slice for example; Fig. $4 \mathrm{~b}$ shows the landmarks identified and marked on the axial slice of Fig. 4a; Fig. 4c is the result of transformation of images into the atlas space; and Fig. $4 \mathrm{~d}$ is a merged view of the PET image and warped atlas image.

Due to the variation of image scanning with different scanners and different parameters, the image intensities need to be normalized before performing the statistical analysis. Since the cerebellum is well preserved in $\mathrm{AD}$, it is selected as the reference region to normalize the other areas including the hippocampus, amygdala, and their adjacent structures. In addition, to reduce the partial volume effect of the connected areas of different brain structures, the pixels with the highest $25 \%$ intensity values and the lowest $25 \%$ intensity values are excluded to calculate the average intensity.

\section{Statistical Analysis}

Fifteen anatomical brain areas are selected as AD-specific structures shown in Table II. They are divided by three different types: 1) the most-affected structures, 2) the adjacent structures of the most-affected structures, and 3) the lessaffected structure by $\mathrm{AD}$. Patients with $\mathrm{AD}$ may have characteristic reductions in glucose metabolic measurements in the structures of the first two types, while the third type structure is the reference for intensity normalization. 
TABLE II. AD-SPECIFIC STRUCTURES

\begin{tabular}{|l|l|l|}
\hline Types & Structure Names & Abbreviations \\
\hline Most-affected & hippocampus & HC \\
\cline { 2 - 3 } & amygdala & AM \\
\cline { 2 - 3 } & interior temporal gyrus & ITG \\
\cline { 2 - 3 } & middle temporal gyrus & MTG \\
\cline { 2 - 3 } & superior temporal gyrus & STG \\
\hline Adjacent & angular gyrus & AG \\
\cline { 2 - 3 } & fusiform gyrus & FG \\
\cline { 2 - 3 } & insular lobe & IL \\
\cline { 2 - 3 } & putamen & PU \\
\cline { 2 - 3 } & globus pallidus lateral & GPL \\
\cline { 2 - 3 } & globus pallidus medial & GPM \\
\cline { 2 - 3 } & parahippocampal gyrus & PG \\
\cline { 2 - 3 } & supramarginal gyrus & SG \\
\cline { 2 - 3 } & thalamus & TH \\
\hline Less-affected & cerebellum & CB \\
\hline
\end{tabular}

The statistical analysis is based on the GTL cases. It includes: 1) the correlation validation of cognitive scores with the AD-specific structures as well as the other variables like age and gender; 2) the stepwise regressions of cognitive scores based on the variables with top correlative values; and 3) discriminant classification of $\mathrm{AD}, \mathrm{MCI}$, and normal subject. The above statistical models are calculated by the Statistical Package for Social Sciences (SPSS) [27] version 16.0. The regression equations and classification functions are validated with the GVD cases by substituting the normalized average values of $A D$-specific structures into them to calculate the regressed cognitive scores and classified results.

\section{1) Data Table}

A data table is created to include all relevant information together for easier calculation by SPSS. The variables in the table consist of four types: 1) personal information including case ID, gender, and age; 2) cognitive scores including MMSE and CDR; 3) diagnosis results; and 4) the normalized average glucose metabolism of all AD-specific structures. An example of AD case is shown at the right-most column of Table III. It is also used as an example for illustration of cognitive assessment and diagnosis classification in the subsequent sections. This 69.7 years old man was diagnosed as AD patient (diagnosis value is 1 ) at the day of study. His MMSE score was only 15 and his CDR score was as high as 2 at the study day. The glucose consumption values of several structures such as the $P G, A M$, and $H C$ have obvious reduction comparing to those of other structures like the $P U$ (see Table II for abbreviations).

\section{2) Correlation Validation}

The correlation test of all variables in Table III is based on bivariate correlation by calculating the Pearson's productmoment coefficient [27]. It is widely used in testing the linear dependence between two variables by giving a value between 1 and +1 , and typically denoted by $R$. It is defined as the covariance of the two variables divided by the product of their standard deviations. The variables with higher $R$ values, and also significant to MMSE and CDR, are used as the independent variable for the stepwise regressions for MMSE and CDR.

\section{3) Regressions of Cognitive Scores}

The purpose of stepwise regressions of MMSE and CDR is to generate the regression equations, which would be used to estimate the cognitive scores for any existing or new case(s). The regression operation in SPSS needs to define one dependent variable and several independent variables. The dependent variable for MMSE regression is MMSE score, and the dependent variable for CDR regression is CDR score. The independent variables are those most correlated structures selected from the AD-specific structures by the previous step "Correlation Validation".

TABLE III. DATA STRUCTURE WITH DESCRIPTIONS OF GTL CASES

\begin{tabular}{|c|c|c|c|}
\hline $\begin{array}{c}\text { Variable } \\
\text { Types }\end{array}$ & Variables & Descriptions & Example \\
\hline \multirow{3}{*}{$\begin{array}{c}\text { Personal } \\
\text { Information }\end{array}$} & CaseID & Unique ID for each subject & 104005 \\
\hline & Gender & Male (1) or Female (2) & 1 \\
\hline & Age & Age at the day of study & 69.7 \\
\hline \multirow{2}{*}{$\begin{array}{l}\text { Cognitive } \\
\text { Scores }\end{array}$} & MMSE & Score at the day of study & 15 \\
\hline & CDR & Score at the day of study & 2 \\
\hline Diagnosis & Diagnosis & $\mathrm{AD}(1), \mathrm{MCI}(2)$, or NL (3) & 1 \\
\hline \multirow{14}{*}{$\begin{array}{c}\text { Average } \\
\text { Glucose } \\
\text { Metabolism }\end{array}$} & $\mathrm{AM}$ & \multirow{14}{*}{$\begin{array}{l}\text { Normalized average } \\
\text { intensity values } \\
\text { to the cerebellum }\end{array}$} & 0.58 \\
\hline & $\mathrm{AG}$ & & 0.65 \\
\hline & $\mathrm{FG}$ & & 0.71 \\
\hline & GPL & & 0.86 \\
\hline & GPM & & 0.84 \\
\hline & $\mathrm{HC}$ & & 0.58 \\
\hline & ITG & & 0.64 \\
\hline & IL & & 0.87 \\
\hline & MTG & & 0.78 \\
\hline & PG & & 0.50 \\
\hline & $\mathrm{PU}$ & & 1.19 \\
\hline & STG & & 0.79 \\
\hline & $\mathrm{SG}$ & & 0.71 \\
\hline & $\mathrm{TH}$ & & 0.63 \\
\hline
\end{tabular}

The stepwise regression repeatedly chooses the predicting variables (one or more of correlated structures) automatically to generate the regression equations by taking a sequence of analysis of variance (ANOVA) test for the equation and t-test for the independent variables. The outcome of the stepwise regression is a list of coefficients corresponding to the predicting variables, and a constant as well. Those coefficients and the constant construct a linear equation for MMSE, if the dependent variable is MMSE score. The CDR regression equation can be generated in the same way.

\section{4) Classification of $A D, M C I$ and $N L$}

Discriminant analysis builds a predictive model for group membership. The model is composed of discriminant functions based on linear combinations of the predictor variables that provide the best discrimination between the groups. The functions are generated from a sample of cases for which group membership is known. The functions can then be applied to new cases that have measurements for the predictor variables but have unknown group membership.

To classify the cases into the groups of $\mathrm{AD}, \mathrm{MCI}$, and NL, the Fisher's linear discriminant analysis [28] is applied to generate the discriminant functions by calculating the Fisher's coefficients of all predicting variables. For discriminant classification in SPSS, the grouping variable is the diagnosis results, i.e. $\mathrm{AD}, \mathrm{MCI}$, and $\mathrm{NL}$. In order to determine whether the most-affected structures by AD are supposed to be included as the predicting variables for Fisher's linear discriminant analysis, two situations are considered: 1) only use the 
cognitive scores as a single input (Method A); and 2) use both the cognitive scores and the normalized average intensity values of the correlated structures as the input (Method B). The output of discriminant classification analysis contains several discriminant function coefficients that can be used directly for classification. As the grouping variable has three values (AD, MCI, and NL), three sets of coefficients of predicting variables are obtained for each group. The testing case is assigned to the group for which it has the largest discriminant score.

\section{5) Result Verification}

The regression equations and discriminant functions obtained by the statistical models need to be verified by all the GTL cases as well as the GVD cases. It is done by substituting the normalized average values of $\mathrm{AD}$-specific structures into the regression equations to calculate the regressed cognitive scores, and into the discriminant functions to classify the testing cases into different diagnosis groups of $\mathrm{AD}, \mathrm{MCI}$, and NL.

For each testing case, the normalized average intensity values of $\mathrm{AD}$-specific structures are the input for both stepwise regression and discriminant classification. They are applied to calculate the regressed values of MMSE and CDR first for cognitive assessment. Thereafter, the discriminant values are computed based on the regresses values. To measure the accuracy of the classification methods, a success rate is defined to quantify for every statistical method, every group of cases, and every category of subjects.

\section{6) Success Rate}

The success rate is defined as a ratio of the number of successful classified cases automatically by the number of cases manually diagnosed in that category.

$$
\operatorname{SuccessRate}(m, c, s)=\frac{\mathrm{A}(m, c, s)}{\mathrm{N}(c)}
$$

where $m$ is the method used (Method A or Method B), $c$ is the subject category (AD, MCI, or NL), $s$ is the score system (MMSE or CDR), $\mathrm{A}(m, c, s)$ is the number of cases which are classified as the category $c$ by the method $\mathrm{m}$ with score system $s . \mathrm{N}(c)$ is the number of cases which are classified manually as the category $c$. For example, if there are $38 \mathrm{AD}$ cases in the group GTL, and the number of cases which were classified as AD by Method A is 29, the success rate of Method A for AD diagnosis is $29 / 38$, i.e. $76.3 \%$

\section{RESULTS}

The proposed approach of automatic cognitive assessment and AD diagnosis by statistical analysis of PET image processing was implemented in $\mathrm{C}++$ running on a standard personal computer with $2.4 \mathrm{G}$ CPU. The computer has the statistical package SPSS version 16.0 installed. The average processing time was 12.4 seconds per case, including the image data processing as well as the calculation of regression equations and discriminant functions. A total of 400 cases with a resolution of $160 \times 160 \times 96$ voxels of size $1.5 \mathrm{~mm}^{3}$ were randomly divided into two groups of GTL and GVD for generating and testing regression equations and discriminant functions, respectively.
TABLE IV. IMAGE PROCESSING RESULTS

\begin{tabular}{|c|c|c|c|c|c|c|}
\hline \multirow{3}{*}{ Name } & \multicolumn{2}{|c|}{$\begin{array}{c}\text { Average and normalized } \\
\text { values (mean } \pm \text { SD) }\end{array}$} & \multicolumn{3}{c|}{$\begin{array}{c}\text { Significances } \\
(\boldsymbol{p})\end{array}$} \\
\cline { 2 - 7 } & $\boldsymbol{A D}$ & $\boldsymbol{M C I}$ & $\boldsymbol{N} \boldsymbol{L}$ & $\begin{array}{c}\boldsymbol{A D} / \\
\boldsymbol{M C I}\end{array}$ & $\begin{array}{c}\boldsymbol{M C I} / \\
\boldsymbol{N L}\end{array}$ & $\begin{array}{c}\boldsymbol{A D} / \\
\boldsymbol{N} \boldsymbol{I}\end{array}$ \\
\hline AM & $0.7 \pm 0.1$ & $0.8 \pm 0.1$ & $0.9 \pm 0.1$ & $<0.001$ & $<0.001$ & $<0.001$ \\
\hline AG & $0.8 \pm 0.2$ & $1.0 \pm 0.2$ & $1.1 \pm 0.2$ & $<0.001$ & $<0.001$ & $<0.001$ \\
\hline FG & $0.8 \pm 0.1$ & $0.8 \pm 0.1$ & $0.9 \pm 0.1$ & 0.333 & $<0.001$ & $<0.001$ \\
\hline GPL & $0.8 \pm 0.1$ & $0.8 \pm 0.1$ & $0.8 \pm 0.2$ & 0.061 & $<0.001$ & $<0.001$ \\
\hline GPM & $0.8 \pm 0.1$ & $0.8 \pm 0.1$ & $0.9 \pm 0.2$ & 0.896 & $<0.001$ & 0.009 \\
\hline HC & $0.7 \pm 0.1$ & $0.8 \pm 0.1$ & $0.9 \pm 0.1$ & $<0.001$ & $<0.001$ & $<0.001$ \\
\hline ITG & $0.6 \pm 0.2$ & $0.6 \pm 0.2$ & $0.7 \pm 0.2$ & 0.655 & $<0.001$ & $<0.001$ \\
\hline IL & $0.9 \pm 0.1$ & $0.9 \pm 0.1$ & $1.1 \pm 0.1$ & 0.002 & $<0.001$ & $<0.001$ \\
\hline MTG & $0.8 \pm 0.2$ & $0.9 \pm 0.1$ & $1.0 \pm 0.1$ & $<0.001$ & $<0.001$ & $<0.001$ \\
\hline PG & $0.6 \pm 0.1$ & $0.6 \pm 0.1$ & $0.7 \pm 0.1$ & 0.002 & $<0.001$ & $<0.001$ \\
\hline PU & $1.1 \pm 0.2$ & $1.1 \pm 0.2$ & $1.2 \pm 0.2$ & 0.075 & $<0.001$ & $<0.001$ \\
\hline STG & $0.9 \pm 0.1$ & $0.9 \pm 0.1$ & $1.0 \pm 0.1$ & 0.001 & $<0.001$ & $<0.001$ \\
\hline SG & $0.8 \pm 0.2$ & $0.9 \pm 0.2$ & $1.0 \pm 0.2$ & $<0.001$ & 0.007 & $<0.001$ \\
\hline TH & $0.8 \pm 0.2$ & $0.9 \pm 0.2$ & $1.0 \pm 0.2$ & 0.001 & 0.001 & $<0.001$ \\
\hline
\end{tabular}

TABLE V. CORRELATION TEST RESULTS

\begin{tabular}{|c|c|c|c|c|c|}
\hline \multirow{2}{*}{ Variables } & \multicolumn{2}{|c|}{ MMSE } & \multirow{2}{*}{ Variables } & \multicolumn{2}{|c|}{ CDR } \\
\cline { 6 - 7 } & $\boldsymbol{R}$ & $\boldsymbol{p}$ & & $\boldsymbol{R}$ & $\boldsymbol{p}$ \\
\hline AM & 0.608 & $<0.001$ & AM & -0.591 & $<0.001$ \\
\hline MTG & 0.596 & $<0.001$ & MTG & -0.566 & $<0.001$ \\
\hline HC & 0.578 & $<0.001$ & IL & -0.555 & $<0.001$ \\
\hline AG & 0.547 & $<0.001$ & HC & -0.497 & $<0.001$ \\
\hline STG & 0.543 & $<0.001$ & PG & -0.496 & $<0.001$ \\
\hline IL & 0.491 & $<0.001$ & STG & -0.490 & $<0.001$ \\
\hline SG & 0.472 & $<0.001$ & AG & -0.457 & $<0.001$ \\
\hline PG & 0.445 & $<0.001$ & SG & -0.406 & $<0.001$ \\
\hline TH & 0.437 & $<0.001$ & TH & -0.356 & $<0.001$ \\
\hline PU & 0.328 & $<0.001$ & FG & -0.329 & $<0.001$ \\
\hline GPM & 0.243 & $<0.001$ & PU & -0.328 & $<0.001$ \\
\hline FG & 0.238 & $<0.001$ & ITG & -0.308 & $<0.001$ \\
\hline ITG & 0.238 & $<0.001$ & GPM & -0.235 & $<0.001$ \\
\hline GPL & 0.110 & 0.083 & GPL & -0.212 & 0.001 \\
\hline Gender & -0.087 & 0.170 & Age & 0.110 & 0.083 \\
\hline Age & -0.124 & 0.05 & Gender & 0.031 & 0.629 \\
\hline
\end{tabular}

\section{1) Data Image Processing}

Table IV lists the results of image data processing, including the normalized glucose metabolism values for all 14 AD-specific structures (the cerebellum is excluded due to its role of reference for normalization). The mean values with the standard deviations (SD) are shown for different groups AD, $\mathrm{MCI}$, and NL, respectively. Based on t-test of independent samples, there are significant differences in several variables, e.g. the amygdala $(\mathrm{AM})$, between the groups of $\mathrm{AD} / \mathrm{MCI}$, $\mathrm{MCI} / \mathrm{NL}$, and $\mathrm{AD} / \mathrm{NL}$, shown in Table IV. Also, several variables like the globus pallidus medial (GPM) do not have significant differences between the groups of $\mathrm{AD} / \mathrm{MCI}$, even though the significant differences are shown between the groups of $\mathrm{MCI} / \mathrm{NL}$ and $\mathrm{AD} / \mathrm{NL}$.

\section{2) Correlation Validation}

In order to validate the correlations between the change of brain structures and the cognitive scores, Table $\mathrm{V}$ lists the Pearson correlation $(R)$ and significant values $(p)$ for both MMSE and CDR by descending sort of $R$. It shows that the variables gender and age are not significant correlated with the cognitive scores; and the other variables of brain structures are more or less correlated with the scores. For more detailed analysis, the top eight common variables of both MMSE and 
CDR in Table $\mathrm{V}$ are chosen as the most correlated brain structures with cognitive scores MMSE and CDR. They are $A M, M T G, H C, A G, S T G, I L, S G$, and $P G$.

\section{3) Regressions of Cognitive Scores}

The stepwise regressions for MMSE and CDR are shown in Table VI. Their progresses are stopped after five and four steps of regressions for MMSR and CDR, respectively. This means the stepwise regressions have five/four steps to enter one predicting variable by each step. In Table VI, $\mathrm{R}^{2}$ is simply the square of the sample correlation coefficient between the outcomes and their predicted values. Adjusted $\mathrm{R}^{2}$ is a modification of $\mathrm{R}^{2}$ that adjusts for the number of explanatory terms in a model. Unlike $\mathrm{R}^{2}$, the adjusted $\mathrm{R}^{2}$ increases only if the new term improves the model more than would be expected by chance. The adjusted $\mathrm{R}^{2}$ can be negative, and will always be less than or equal to $\mathrm{R}^{2}$.

TABLE VI. STEPWISE REGRESSIONS OF MMSE AND CDR

\begin{tabular}{|l|c|c|c|c|c|}
\hline & Steps & $\mathbf{R}^{\mathbf{2}}$ & Adjusted $^{\mathbf{2}}$ & $\boldsymbol{F}$ & $\boldsymbol{p}$ \\
\hline MMSE & 1 & 0.370 & 0.368 & 145.775 & $<0.001$ \\
\cline { 2 - 6 } & 2 & 0.474 & 0.469 & 111.110 & $<0.001$ \\
\cline { 2 - 6 } & 3 & 0.526 & 0.520 & 90.924 & $<0.001$ \\
\cline { 2 - 6 } & 4 & 0.543 & 0.535 & 72.699 & $<0.001$ \\
\cline { 2 - 6 } & 5 & 0.554 & 0.545 & 60.712 & $<0.001$ \\
\hline CDR & 1 & 0.349 & 0.347 & 133.208 & $<0.001$ \\
\cline { 2 - 6 } & 2 & 0.438 & 0.433 & 96.070 & $<0.001$ \\
\cline { 2 - 6 } & 3 & 0.456 & 0.449 & 68.647 & $<0.001$ \\
\cline { 2 - 6 } & 4 & 0.474 & 0.465 & 55.170 & $<0.001$ \\
\hline
\end{tabular}

TABLE VII. STEPWISE REGRESSION EQUATIONS OF MMSE AND CDR

\begin{tabular}{|c|c|c|c|c|}
\hline & Variables & Coefficients & t-values & Significances (p) \\
\hline \multirow{4}{*}{ MMSE } & $\mathrm{AM}$ & 11.574 & 6.097 & $<0.001$ \\
\cline { 2 - 5 } & $\mathrm{MTG}$ & 13.533 & 7.742 & $<0.001$ \\
\cline { 2 - 5 } & $\mathrm{AG}$ & 7.673 & 6.485 & $<0.001$ \\
\cline { 2 - 5 } & $\mathrm{STG}$ & -8.144 & -2.745 & 0.007 \\
\cline { 2 - 5 } & $\mathrm{IL}$ & -5.328 & -2.525 & 0.012 \\
\cline { 2 - 5 } & (Constant) & 10.221 & 6.788 & $<0.001$ \\
\hline \multirow{4}{*}{$\mathrm{CDR}$} & $\mathrm{AM}$ & -1.338 & -5.973 & $<0.001$ \\
\cline { 2 - 5 } & $\mathrm{MTG}$ & -1.462 & -6.573 & $<0.001$ \\
\cline { 2 - 5 } & $\mathrm{AG}$ & -0.611 & -3.961 & $<0.001$ \\
\cline { 2 - 5 } & $\mathrm{STG}$ & 1.135 & 2.912 & 0.004 \\
\cline { 2 - 5 } & (Constant) & 2.391 & 12.681 & $<0.001$ \\
\hline
\end{tabular}

TABLE VIII. DISCRIMINANT FUNCTION COEFFICIENTS

\begin{tabular}{|c|c|c|c|c|c|}
\hline Scores & Methods & Variables & AD & MCI & NL \\
\hline \multirow{6}{*}{ MMSE } & \multirow{2}{*}{ Method A } & $\mathrm{R}_{\mathrm{MMSE}}$ & 6.53 & 7.256 & 8.01 \\
\hline & & (Constant) & -76.95 & -94.79 & -115.27 \\
\hline & \multirow{4}{*}{ Method B } & $\mathrm{R}_{\mathrm{MMSE}}$ & 5.25 & 6.00 & 6.48 \\
\hline & & IL & 51.91 & 49.97 & 57.14 \\
\hline & & PG & 8.36 & 9.54 & 17.60 \\
\hline & & (Constant) & -87.63 & -105.02 & -130.52 \\
\hline \multirow{7}{*}{ CDR } & \multirow{2}{*}{ Method A } & $\mathrm{R}_{\mathrm{CDR}}$ & 18.55 & 12.43 & 4.65 \\
\hline & & (Constant) & -8.48 & -4.42 & -1.56 \\
\hline & \multirow{5}{*}{ Method B } & $\mathrm{R}_{\mathrm{CDR}}$ & 126.27 & 121.67 & 117.30 \\
\hline & & $\mathrm{AG}$ & 112.51 & 119.10 & 120.62 \\
\hline & & $\mathrm{IL}$ & 125.35 & 117.53 & 122.06 \\
\hline & & PG & 165.20 & 171.39 & 179.79 \\
\hline & & (Constant) & -198.01 & -197.10 & -207.10 \\
\hline
\end{tabular}

Table VII lists the coefficients for each predicting variable as well as the constants of regression equations. For example, the MMSE regressions have five predicting variables: $A M$,
$M T G, A G, S T G$, and $I L$. The linear regression equation for calculation of cognitive MMSE scores $\left(R_{m}\right)$ is constructed from these coefficients and the constant.

$R_{m}=11.574 \times A M+13.533 \times M T G+7.673 \times A G-8.144 \times S T G-5.328 \times I L+10.221$

For CDR, the linear regression equation for calculation of CDR scores $\left(R_{c}\right)$ is similarly constructed from four coefficients of predicting variables $A M, M T G, A G$, and $S T G$.

$R_{c}=-1.338 \times A M-1.462 \times M T G-0.611 \times A G+1.135 \times S T G+2.391$

For the example in Table III, $A M=0.58, M T G=0.78$, $A G=0.65, S T G=0.79$, and $I L=0.87$, the calculation results of regressed MMSE $\left(R_{m}\right)$ and regressed $\mathrm{CDR}\left(R_{c}\right)$ are 21.41 and 0.97 by equations (1) and (2), respectively.

\section{4) Classification of AD, MCI and Normal}

There is only one predicting variable for Method A. It is regressed MMSE $\left(R_{m}\right)$ or regressed $\mathrm{CDR}\left(R_{c}\right)$ for MMSE scores or CDR scores as shown in Table VIII. For Method B, there are two additional predicting variables $I L$ and $P G$ for MMSE scores and three additional predicting variables $A G, I L$, and $P G$ for CDR scores. Each variable has its coefficient to construct discriminant functions with a constant for each function. The coefficients of the predicting variables and the constants are listed in Table VIII. For each method, there are three functions to discriminate one group from others. For example, the functions of MMSE for Method A are described by equation (3), and the functions for Method B are described by equation (4).

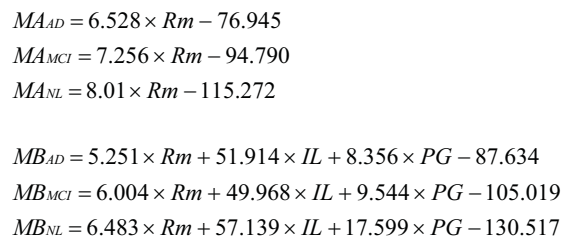

The functions of CDR for Method A are described by equation (5), and the functions for Method B are described by equation (6).

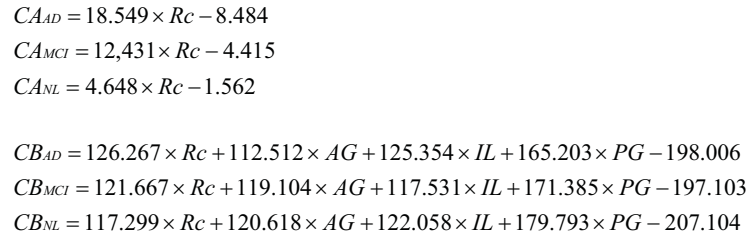

For the case in Table III $I L$ is $0.87, P G$ is 0.50 , and $R m$ is 21.41, equation (4) gives the results of Method B: $M B_{A D}=74.19, \quad M B_{M C I}=71.77$, and $M B_{N L}=61.65$. As the maximum value of three variables is $M B_{A D}$, the subject is classified as a patient with AD.

\section{5) Result Verification}

The final classification results are given in Table IX, including 250 GTL cases and 150 GVD cases. For GTL cases, 
the average success rates of Method A are $62.8 \%$ and $62.4 \%$ by the regressed-MMSE and regressed-CDR, respectively; the average success rates of Method B are $65.6 \%$ and $69.2 \%$ correspondingly. For GVD cases, the success rates of Method A are $52 \%$ and $56.7 \%$, and those of Method B are $61.3 \%$ and $60.7 \%$.

TABLE IX. ClasSIFICATION RESULTS (SUCCESS RATE)

\begin{tabular}{|c|c|c|c|c|c|c|}
\hline Grps & Scores & Methods & $\begin{array}{l}\text { AD } \\
(\%)\end{array}$ & $\begin{array}{c}\text { MCI } \\
(\%)\end{array}$ & $\begin{array}{l}\text { NL } \\
(\%)\end{array}$ & $\begin{array}{l}\text { Total } \\
\text { (\%) }\end{array}$ \\
\hline \multirow{5}{*}{ GTL } & \multicolumn{2}{|c|}{ Number of Cases } & 38 & 133 & 79 & 250 \\
\hline & \multirow{2}{*}{ MMSE } & Method A & $\begin{array}{c}29 \\
(76.3)\end{array}$ & $\begin{array}{c}64 \\
(48.1)\end{array}$ & $\begin{array}{c}64 \\
(81.0)\end{array}$ & $\begin{array}{c}157 \\
(62.8)\end{array}$ \\
\hline & & Method B & $\begin{array}{c}28 \\
(73.7)\end{array}$ & $\begin{array}{c}73 \\
(54.9)\end{array}$ & $\begin{array}{c}63 \\
(79.7)\end{array}$ & $\begin{array}{c}164 \\
(65.6)\end{array}$ \\
\hline & \multirow{2}{*}{ CDR } & Method A & $\begin{array}{c}28 \\
(73.7)\end{array}$ & $\begin{array}{c}64 \\
(48.1)\end{array}$ & $\begin{array}{c}64 \\
(81.0)\end{array}$ & $\begin{array}{c}156 \\
(62.4)\end{array}$ \\
\hline & & Method B & $\begin{array}{c}32 \\
(84.2)\end{array}$ & $\begin{array}{c}77 \\
(57.9)\end{array}$ & $\begin{array}{c}64 \\
(81.0)\end{array}$ & $\begin{array}{c}173 \\
(69.2)\end{array}$ \\
\hline \multirow{5}{*}{ GVD } & \multicolumn{2}{|c|}{ Number of Cases } & 28 & 79 & 43 & 150 \\
\hline & \multirow{2}{*}{ MMSE } & Method A & $\begin{array}{c}19 \\
(67.9)\end{array}$ & $\begin{array}{c}35 \\
(44.3)\end{array}$ & $\begin{array}{c}24 \\
(55.8)\end{array}$ & $\begin{array}{c}78 \\
(52.0)\end{array}$ \\
\hline & & Method B & $\begin{array}{c}19 \\
(67.9)\end{array}$ & $\begin{array}{c}47 \\
(59.5)\end{array}$ & $\begin{array}{c}26 \\
(60.5)\end{array}$ & $\begin{array}{c}92 \\
(61.3)\end{array}$ \\
\hline & \multirow{2}{*}{ CDR } & Method A & $\begin{array}{c}20 \\
(71.4)\end{array}$ & $\begin{array}{c}42 \\
(53.2)\end{array}$ & $\begin{array}{c}23 \\
(53.5)\end{array}$ & $\begin{array}{c}85 \\
(56.7)\end{array}$ \\
\hline & & Method B & $\begin{array}{c}19 \\
(67.9)\end{array}$ & $\begin{array}{c}47 \\
(59.5)\end{array}$ & $\begin{array}{c}25 \\
(58.1)\end{array}$ & $\begin{array}{c}91 \\
(60.7)\end{array}$ \\
\hline
\end{tabular}

There are several observations on the classification results of Table IX. More discussions are given in the Discussion section.

- The success rates of Method B are higher than those of Method A for both MMSE and CDR scores and for both groups of GTL and GVD as well.

- The success rates of MCI are much lower than those of $\mathrm{AD}$ and NL in each method and each group of cases.

- The success rates of GVD cases are lower than those of GTL cases.

- The success rates of different score systems show no major difference. In other words, both MMSE and CDR are appropriate to present the cognition impairment

\section{DISCUSSION}

Numerous algorithms of medical image segmentation were implemented and presented for both structural medical images like MRI or CT, and functional medical images like PET. However, the segmentation of ROIs from PET neuroimages faces more difficulties due to the absence of boundary information in these images. Accurate segmentation of a particular structure from PET images automatically is almost not possible. Therefore, atlas-based methods have their advantages to identify several landmarks instead of the identification of the boundaries. The accuracy of atlas-based methods highly depends on that of landmark identification. The inaccurate landmark identification causes the inaccurate information extracted from the ROI, i.e. the measurement of average glucose metabolism in our research work. It is a very time-consuming task to ensure the accuracy of the landmark identification by visual inspection case by case by neurological or radiological experts.
After the fast and automatic data transformation of PET images into the atlas space, the information extracted from the ROIs of hundreds of cases is performed by several statistical models such as correlation validation, stepwise regression, and discriminant classification. There are several observations on the statistical results.

\section{a) Method B is superior to Method $A$}

In average, the success rates of Method B are higher than those of Method A. That means the correlated structures are also the important factors for the discriminant classification even through the regressed cognitive scores are already based on these structures.

b) Success rates of $M C I$

Although the experiment data have three category groups: $\mathrm{AD}, \mathrm{MCI}$, and NL, most of the $\mathrm{AD}$ cases are at mild stage. There is only a few cases $(<1 \%)$ are at moderate stage, and no severe AD cases at all. If the changes of glucose metabolism on the AD-specific structures are too subtle to be distinguished by the classification algorithm, it is a high possibility to classify the MCI cases into AD or NL. In addition, the calculation of success rates is based on the clinical diagnosis results, which may not only depend on the cognitive scores, but also the experience of doctors. There may have some deviation between the doctor's judgment and the cognitive score. The conflicts between the cognitive scores and the diagnosis results are found in several cases. For example, a patient with $27 \mathrm{MMSE}$ score was diagnosed as AD, and another case with $25 \mathrm{MMSE}$ score was classified as normal subject. For the category of MCI, the highest MMSE score of MCI patients is 30 and the lowest is only 19, but both of them were diagnosed as MCI.

\section{c) Success rates of GVD cases}

The statistical models are performed on the GTL cases, which have higher success rates than those of GVD cases. The regression equations and discriminant functions are based on an assumption that the cognitive scores such as MMSE and CDR have linear correlation with the changes of glucose metabolism on AD-specific structures. If the assumption is not always true, or the cognitive scores have correlated with the structures other than those we defined as AD-specific, the regression equations or discriminant functions may depend highly on the template data. When they are applied to the other data which are not the part of template cases, the success rates are dropped.

d) AD-affected structures are excluded from the statistical analysis

The hippocampus and parahippocampal gyrus are considered the firstly affected structures in the brain by AD. However, they are too small to be accurately segmented by the automatic computer algorithms. In addition, the partial volume effect may be more serious in those small structures than the larger structures due to the high ratio of boundaries by the whole structure areas.

In summary, the results of statistical analysis depend on several factors like the number of samples, statistical models, data distributions, etc. There are a few possible ways to increase the success rates of stepwise regressions by: 1) adding more brain structures as the independent variables; 2) removing 
the noise data, for example, the cases with low MMSE but was diagnosed as MCI or NL and vice versa; 3) separating the left and right hemispheres for each brain structure; and 4) exploring other statistical models such as neural network and nonparameter tests.

\section{CONCLUSION}

In conclusion, this paper presents an automated atlasassisted approach, which makes use of the image data processing methods and a high resolution brain atlas, together with the statistical analysis of stepwise regressions and discriminant classification to assess the cognitive scores and distinguish the patients with $\mathrm{AD}$ or MCI from normal subjects. The approach has been applied to hundreds of cases and shown promising results. For template cases, the average success rates are $65.6 \%$ and $69.2 \%$ by Method B with MMSE scores and CDR scores, respectively. For validation cases, the average success rates are $61.3 \%$ and $60.7 \%$ by Method B. In spite of the low success rates for MCI diagnosis, we believe that our methods are useful by testing on more cases and trying other statistical models in the future.

\section{ACKNOWLEDGMENT}

The data for this study have been obtained from the Alzheimer's Disease Neuroimaging Initiative (ADNI) database (www.loni.ucla.edu\ADNI). This research is partially supported by ARC DP0773584.

\section{REFERENCES}

[1] R. Petersen, G. Smith, S. Waring, R. Ivnik, E. Tangalos, and E. Kokmen, "Mild cognitive impairment: clinical characterization and outcome," Archives of neurology, vol. 56, p. 303, 1999.

[2] M. Folstein, S. Folstein, and P. McHugh, "Mini-Mental State: a practical method for grading the cognitive state of patients for the clinician," J Psychiatr Res, vol. 12, pp. 189-198, 1975.

[3] J. Morris, "The Clinical Dementia Rating (CDR): current version and scoring rules," Neurology, vol. 43, pp. 2412-2414, 1993.

[4] C. R. Jack, D. W. Dickson, J. E. Parisi, Y. C. Xu, R. H. Cha, P. C. O'Brien, S. D. Edland, G. E. Smith, B. F. Boeve, and E. G. Tangalos, "Antemortem MRI findings correlate with hippocampal neuropathology in typical aging and dementia," Neurology, vol. 58, pp. 750-757, 2002.

[5] P. Mouton, L. Martin, M. Calhoun, G. Dal Forno, and D. Price, "Cognitive decline strongly correlates with cortical atrophy in Alzheimer's dementia," Neurobiology of Aging, vol. 19, pp. 371-377, 1998.

[6] M. Kawano, A. Ichimiya, K. Ogomori, Y. Kuwabara, M. Sasaki, T. Yoshida, and N. Tashiro, "Relationship between both IQ and MiniMental State Examination and the regional cerebral glucose metabolism in clinically diagnosed Alzheimer's disease: a PET study," Dementia and geriatric cognitive disorders, vol. 12, pp. 171-176, 2000.

[7] A. Osawa, S. Maeshima, Y. Shimamoto, E. Maeshima, E. Sekiguchi, K. Kakishita, F. Ozaki, and H. Moriwaki, "Relationship between cognitive function and regional cerebral blood flow in different types of dementia," Disability \& Rehabilitation, vol. 26, pp. 739-745, 2004.

[8] L. Mosconi, "Brain glucose metabolism in the early and specific diagnosis of Alzheimer's disease," European Journal of Nuclear Medicine and Molecular Imaging, vol. 32, pp. 486-510, 2005.

[9] M. Borrie, "Functional neuroimaging in the diagnosis of dementia," Alzheimer's \& Dementia: The Journal of the Alzheimer's Association, vol. 3, pp. 336-340, 2007.

[10] J. M. Hoffman, K. A. Welsh-Bohmer, M. Hanson, B. Crain, C. Hulette, N. Earl, and R. E. Coleman, "FDG PET Imaging in Patients with Pathologically Verified Dementia," J Nucl Med, vol. 41, pp. 1920-1928, November 1, 20002000.
[11] M. E. Phelps, J. C. Mazziotta, and H. R. Schelbert, Positron Emission Tomography and Autoradiography: Principles and Applications for the Brain and Heart: New York, 1986.

[12] K. Friston, J. Ashburner, S. Kiebel, T. Nichols, and W. Penny, "Statistical parametric mapping: the analysis of functional brain images," Academic Press, Nov 2006.

[13] S. M. Smith, M. Jenkinson, M. W. Woolrich, C. F. Beckmann, T. E. J. Behrens, H. Johansen-Berg, P. R. Bannister, M. De Luca, I. Drobnjak, and D. E. Flitney, "Advances in functional and structural MR image analysis and implementation as FSL," Neuroimage, vol. 23, pp. 208-219, 2004.

[14] C. R. Jack, R. C. Petersen, Y. Xu, P. C. O'Brien, G. E. Smith, R. J Ivnik, B. F. Boeve, E. G. Tangalos, and E. Kokmen, "Rates of hippocampal atrophy correlate with change in clinical status in aging and AD," Neurology, vol. 55, pp. 484-490, 2000.

[15] J. McColl, A. Holmes, and I. Ford, "Statistical methods in neuroimaging with particular application to emission tomography," Statistical methods in medical research, vol. 3, p. 63, 1994.

[16] W. L. Nowinski, G. Qian, K. N. Bhanu Prakash, Q. Hu, and A. Aziz, "Fast Talairach Transformation for magnetic resonance neuroimages," Journal of computer assisted tomography, vol. 30, p. 629, 2006.

[17] W. L. Nowinski and D. Belov, "Toward atlas-assisted automatic interpretation of MRI morphological brain scans in the presence of tumor," Academic radiology, vol. 12, pp. 1049-1057, 2005.

[18] W. L. Nowinski, G. Qian, K. N. B. Prakash, I. Volkau, W. K. Leong, S. Huang, A. Ananthasubramaniam, J. Liu, T. T. Ng, and V. Gupta, "Stroke Suite: CAD systems for acute ischemic stroke, hemorrhagic stroke, and stroke in ER," Lecture Notes In Computer Science, vol. 4987, pp. 377-386, 2008

[19] J. Talairach and P. Tournoux, Co-planar stereotaxic atlas of the human brain: 3-dimensional proportional system: an approach to cerebra imaging: Thieme, 1988.

[20] W. L. Nowinski, "Modified Talairach landmarks," Acta Neurochirurgica, vol. 143, pp. 1045-1057, 2001.

[21] G. Qian, S. Luo, J. Jin, and W. L. Nowinski, "Extended Talairach Landmarks on Neuroimages for Atlas Registration," in The 4th International Conference on Bioinformatics and Biomedical Engineering (iCBBE 2010) Chengdu, China, Jun 18-20, 2010.

[22] Y. Ushijima, C. Okuyama, S. Mori, T. Nakamura, T. Kubota, and T. Nishimura, "Relationship between cognitive function and regional cerebral blood flow in Alzheimer's disease," Nuclear medicine communications, vol. 23, p. 779, 2002.

[23] A. Lockwood, K. Weissenborn, M. Bokemeyer, U. Tietge, and W Burchert, "Correlations between cerebral glucose metabolism and neuropsychological test performance in nonalcoholic cirrhotics," Metabolic brain disease, vol. 17, pp. 29-40, 2002.

[24] J. B. S. Langbaum, K. Chen, W. Lee, C. Reschke, D. Bandy, A. S Fleisher, G. E. Alexander, N. L. Foster, M. W. Weiner, and R. A. Koeppe, "Categorical and correlational analyses of baseline fluorodeoxyglucose positron emission tomography images from the Alzheimer's Disease Neuroimaging Initiative (ADNI)," Neuroimage, vol. 45, pp. 1107-1116, 2009

[25] W. Nowinski, B. Chua, G. Qian, I. Volkau, M. Knopp, V. Runge, Y. Marchenko, F. Puspitasari, N. Nowinska, L. Lee, and G. Yang, "The human brain in 1,002 pieces: A comprehensive and interactive $3 \mathrm{D}$ atlas of structure, vasculature, and connections from multiple 3T and 7T acquisitions.," in RSNA 95th Scientific Assembly and Annual Meeting Chicago, Illinois, 2009, p. 1023.

[26] G. Qian, S. Luo, J. Jin, M. Park, and W. L. Nowinski, "A fast and automatic approach to extract the brain and midsagittal lines from FDGPET head scans," in The 1st International Conference on Information Science and Engineering Nanjing, China, Dec 18-20, 2009.

[27] M. Norusis, SPSS 16.0 Guide to Data Analysis: Prentice Hall Press Upper Saddle River, NJ, USA, 2008.

[28] R. Duda and P. Hart, Pattern Classification and Scene Analysis, 1973. 


\title{
Text Knowledge Representation Model based on Human Concept Learning
}

\author{
Xiangfeng $\mathrm{Luo}^{1,2}$, Chuanliang $\mathrm{Cai}^{1,2}$, Qingliang $\mathrm{Hu}^{1}$ \\ ${ }^{1}$ School of Computer Engineering and Science, Shanghai University \\ ${ }^{2}$ High Performance Computing Center, Shanghai University \\ Shanghai 200072, P.R. China \\ \{luoxf, caichuanliang\}@shu.edu.cn,hdhuq1@163.com
}

\begin{abstract}
The essential abilities of text knowledge representation, such as automatic construction, carrying abundant semantics and flexible reasoning, should be held due to the rapid growth of web resources and the requirements of the reasoning-based web services. However, current text knowledge representation models either lose many textual semantics or cannot be constructed automatically. To solve the above issues, text knowledge representation model is proposed based on the concept algebra of human concept learning. Then, the degree-2 power series hypothesis is developed and the reasoning ability of text representation is proposed. Finally, the results compared with current knowledge representation models show that our model performs better than other models in representing text knowledge.
\end{abstract}

Keywords-text representation; power series; human concept learning; machine understanding

\section{INTRODUCTION}

The essential abilities of text knowledge representation, such as automatic construction, carrying abundant semantics and flexible reasoning, should be held due to the rapid growth of web resources (e.g. web pages) and the requirements of the reasoning-based web services.

Generally speaking, there are four main types of knowledge representation models. 1) Statistics model, such as vector space model (VSM), latent semantic analysis (LSA). 2) Cognition based model, such as element fuzzy cognitive map (EFCM), concept algebra based model [1] [2]. 3) Probability topic model, such as author topic mode (ATM) [3], author-recipient-topic model (ART) [4], correlated topic models (CTM) [5]. 4) Ontology based models, such as ontology inference layer (OIL) [6], ontology web language (OWL) [7].

From the analysis of the above models, we know that those models either are complicated computationally (e.g. CTM) or cannot be constructed automatically (e.g. OWL), even lose many textual semantics (e.g. VSM) and lack the ability of flexible reasoning. Herein, a text can be regarded as a concept and sentences contained in text can be regarded as objects belonging to the text, where the keywords are the attributes of these objects [8]. Based on concept algebra [9], power series representation of text knowledge is developed in this paper. The proposed model not only can carry more abundant

This work is supported by the Shanghai Municipal Science and Technology Commission (project no.09JC1406200), the National Science Foundation of China (project no. 90612010), and the Shanghai Leading Academic Discipline Project (J50103).

Proc. 9th IEEE Int. Conf. on Cognitive Informatics [ICCl'10] F. Sun, Y. Wang, J. Lu, B. Zhang, W. Kinsner \& L.A. Zadeh [Eds.] 978-1-4244-8040-1/10/\$26.00 @2010 IEEE semantics from text (e.g. web page) but also can be represented automatically, which adapts to the rapid growth of web resources and holds more abundant semantics than VSM and EFCM.

This paper is organized as follows. The idea of concept algebra of human concept learning will be introduced in section II. In section III, we propose our text representation model base on the concept algebra of human concept learning. Then, the reasoning rules of text knowledge have been discussed in section IV. Finally, comparison results with other models and conclusions are given in section $\mathrm{V}$ and VI, respectively.

\section{Concept Algebra of Human ConcePt LeARning}

\section{A. Concept Algebra}

Feldman pointed out that any set of data exhibits many patterns, some of which may be specified with only a small number of features, while others may be described by a larger number of features and other patterns involve an intermediate number of features to specify [9]. The key issue is how the arbitrary patterns of data can be described by the combinations of component patterns. In order to solve this issue, in the following parts, we will introduce the related work by an example (Fig.1) cited from [9].

The "world" $W$ consisting of the five amoeba-like objects corresponds to a concept of human concept learning. We choose some "language" in which to express the structure of this "world" $W$, which we think of as simply as a list $\Sigma=$ $\left\{\sigma_{1}, \sigma_{2}, \ldots, \sigma_{d}\right\}$ of abstract property tags, called the property

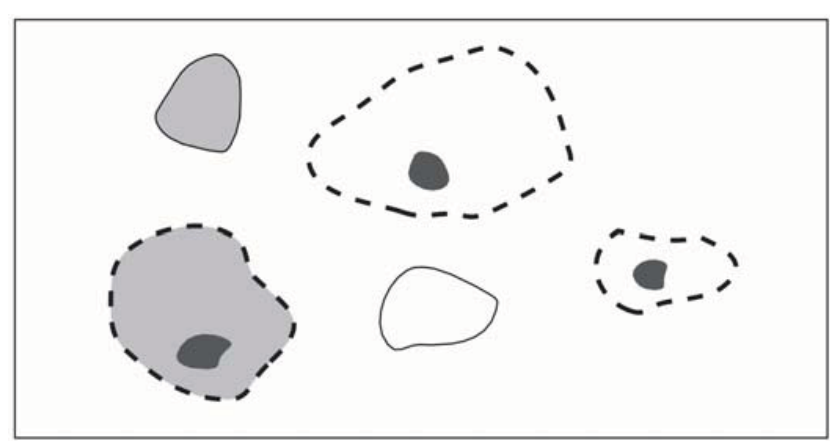

Figure 1. A "world" $W$ containing amoeba-like objects cited from [9]. 